\title{
On Demand Local Link Repair Algorithm for AODV Protocol
}

\author{
Jyoti Jain \\ Dept of Electronics \& Comm. \\ Rajiv Gandhi Technical \\ University \\ BHOPAL, INDIA
}

\author{
Roopam Gupta \\ Dept of Information Tech. \\ Rajiv Gandhi Technical \\ University \\ BHOPAL, INDIA
}

\author{
T.K. Bandhopadhyay \\ Dept of Electronics \& Comm \\ Bansal Institute of Technology \\ BHOPAL, INDIA
}

\begin{abstract}
MANET (Mobile Ad hoc Network) is a wireless self-organized distributed network. This paper gives a general survey of research on local repair of link, if it is broken during communication for MANET and proposes a new local repair scheme in order to make up the deficiency of the existing local repair schemes. The improved local repair scheme concerns about the over head requirement and end to end delay in transmission. Nodes are required to keep the next two-hop node address for each route entry in routing table. During local repair, the repairing node use Ant algorithm for finding new route for next to next node in the link considering that other part of the link is already in existence. Reduced size of F-ANT and B-ANT will give significant reduction in overhead. In this case repairing node not only tries to discover the route to the destination node of data packet, but also attempts to establish the route to its downstream node (i.e. the next hop node). The proposed algorithm will be highly adaptive, scalable and efficient and mainly reduces end-to-end delay in high mobility cases.
\end{abstract}

\section{General Terms}

Mobile Ad hoc Network, AODV protocol

\section{Keywords}

Routing protocol; AODV; link break ; Ant colony; local repair ; Ad hoc mobile networking.

\section{INTRODUCTION}

Mobile ad hoc network (MANET) is the most innovative and challenging area of wireless networking. MANET is an autonomous collection of mobile users that communicate over relatively band width constrained wireless links. Some situations in which user-required infrastructure is not available, cannot be installed, or cannot be installed in time in a given geographic area. In these situations, mobile ad hoc network provides the needed connectivity and network services. With changing technology mobile devices getting smaller, cheaper, more convenient, and more powerful, they also run more applications and network services. All of these factors are fueling the explosive growth of the mobile computing equipment market seen today. New alternative ways to deliver connectivity have been gaining increased attention in recent years. These are focused around having mobile devices within the transmission range connect to each other through automatic configuration, setting up an ad hoc mobile network that is both flexible and powerful. By this way, mobile nodes communicate with each other and receive Internet services through an Internet gateway node, effectively extending both network and Internet services to non infrastructure $[1,2]$.

Characteristics of ad hoc networks include resource-poor devices, limited bandwidth, high error rates, and a continually changing topology. Among the available resources, battery power is typically the most constraining. Hence, routing protocol must have Minimal control overhead, Minimal processing overhead, Multi-hop routing capability, Dynamic topology maintenance and Loop prevention. Many proactive and reactive routing are already in existence [3]. Ad Hoc OnDemand Distance Vector Routing protocol is a reactive protocol. Discussion of this protocol is given in the section 2 . Section 3 will discuss Local link repairing methods and their comparison. Ant algorithm and its application in MANET is given in section 4. New proposed algorithm and protocol is given in section 5. Section 6 will give result and conclusion.

\section{AD HOC ON-DEMAND DISTANCE VECTOR ROUTING}

The Ad Hoc On-Demand Distance Vector (AODV) Routing Protocol [3] provides on-demand route discovery in mobile ad hoc networks. A node does not have to discover and maintain a route to another node until the two needs to communicate. When the local connectivity of the mobile node is of interest, each mobile node can become aware of the other nodes in its neighborhood by the use of several techniques, including local broad-casts known as hello messages. The routing tables of the nodes within the neighborhood are organized to optimize response time to local movements and provide quick response time for requests for establishment of new routes. Primary objectives of this algorithm are [4]:

-To broadcast discovery packets only when necessary.

-To distinguish between local connectivity management, neighborhood detection and general topology maintenance.

-To disseminate information about changes in local connectivity to those neighboring mobile nodes that is likely to need the information.

Route finding in this protocol is based on a route discovery cycle involving a broadcast network search and a unicast reply containing discovered paths. AODV relies on per-node sequence numbers for loop freedom and for ensuring selection of the most recent routing path. AODV nodes maintain a route table in which next-hop routing information for destination nodes is stored. Each routing table entry has an associated lifetime value. If a route is not utilized within the lifetime period, the route is expired. Otherwise, each time the route is used, the lifetime period is updated so that the route is not prematurely deleted. When a source node has data packets to 
send to some destination, it first checks its route table to determine whether it already has a route to the destination. If such a route exists, it can use that route for data packet transmissions. Otherwise, it must initiate a route discovery procedure to find a route. Four types of control packets are used to discover the path in the network if required. As shown in figure 1 [Chakeres and Royer].

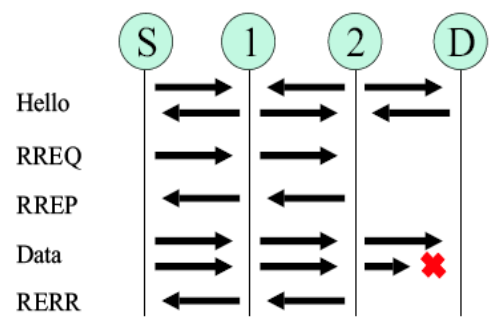

Figure 1 AODV protocol messaging

RREQ (Route request)- This packet is broad-cast by the source node (node which require to communicate with other node ) if it is not having routing information in table. Every node maintains two separate counters, a node sequence number and a broadcast id. The RREQ contains the following fields < source address, source sequence, broadcast-id, dest-address, dest-sequence , hop count $>$ The pair source address \& broadcast- id , uniquely identifies a RREQ. Broadcast-id is incremented whenever the source issues a new RREQ. Each neighbor either satisfies the RREQ by sending a route reply RREP back to the source or re broadcasts the RREQ to its own neighbors after increasing the hop count. Notice that a node may receive multiple copies of the same route broadcast packet from various neighbors. When an intermediate node receives a RREQ, it checks whether it has an unexpired route to the destination. If it does have such a route, then one other condition must hold for the node to generate a reply message indicating the route. The node's route table entry for the destination must have a corresponding sequence number that is at least as great as the indicated destination sequence number in the route request. If intermediate node already received a RREQ with the same broadcast id and source address, it drops the redundant RREQ and does not rebroadcast it. Destination sequence no. is used to find the most recent route, and also guarantees loop freedom.

RREP (Route reply) - Route reply is generated either by destination or by intermediate node, if it is already having a valid route to destination. By the time a broadcast packet arrives at a node that can supply a route to the destination. a reverse path has been established to the source of the RREQ. A RREP contains the Information < source address, dest address, dest sequence, hop count, lifetime $>$. The hop count field in the RREP is set equal to the node's distance from the destination. If the destination itself is creating the RREP, the hop count is set equal to zero. After creating the reply, the node unicasts the message to its next hop toward the source node. Thus, the reverse route that was created as the RREQ was forwarded is utilized to route the RREP back to the source node. When intermediate node receives the RREP, it first creates a forward route entry for the destination node. It uses the node from which it received the RREP as the next hop toward the destination. The hop count for that route is the hop count in the RREP, incremented by one. This forward route entry for the destination will be utilized if the source selects this path for data packet transmissions to the destination.
RERR (route error)- Once a route is established, it must be maintained as long as it is needed. During the communication, if any node in active is moved out of range, links along paths are likely to break. Breaks in active routes must be quickly repaired so that data packets are not dropped [4]. When a link break along an active path occurs, the node upstream of the break (i.e., closer to the source node) invalidates the routes to each of those destinations in its route table. It then creates a route error (RERR) message. In this message it lists all of the destinations that are now unreachable due to the loss of the link. The RERR message traverses the reverse path to the source node. Once the source node receives the RERR, it can repair the route if the route is still needed.

In AODV, routes are maintained as follows: If a source node moves, it has to reinitiate the route discovery protocol to find a new route to the destination. If a node along the route moves, its upstream neighbor notices the move and propagates a link failure notification message (an RREP with an infinite metric) to each of its active upstream neighbors to inform them of the erasure of that part of the route [C K Toh]. These nodes in turn propagate the link failure notification to their upstream neighbors, and so on, until the source node is reached. The source node may then choose to re-initiate route discovery for that destination if a route is still desired.

Hello messages AODVprotocol uses of hello messages which are periodic local broadcasts made by a node to inform each mobile node of other nodes in its neighborhood. Hello messages can be used to maintain the local connectivity of a node. However, the use of hello messages is not required. Nodes listen for retransmissions of data packets to ensure that the next hop is still within reach. If such a retransmission is not heard, the node may use any one of a number of techniques, including the reception of hello messages.

\section{REVIEW OF LOCAL LINK REPAIRING PROTOCOL}

With the help of above mentioned control packets AODV initiate route request on demand by sending RREQ and get available path by RREP. Freshness of route is maintained with the help of sequence number. Error in route is detected by hello message; in case of route error RERR message is generated by the node detecting route failure. Original AODV does not exploit the fast and localized partial route recovery method. It does not have any mechanism to find alternate path (figure 2) in between the transmission link. Many other researchers worked in the direction of route maintenance if it has link breakage during the transmission of data.

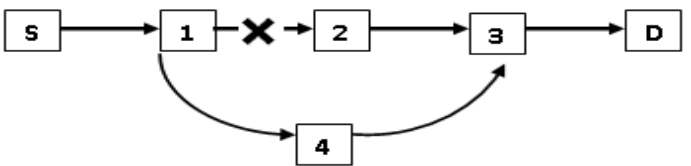

Figure 2 link break during transfer of data

In 2003 Srdjan Krco and Marina Dupcinov proposed Improved Neighbor Detection Algorithm for AODV Routing Protocol for the improvement of link quality [5]. This algorithm is developed to check the quality of link as a function of SNR. Link with better quality factor are selected for the communication, In this algorithm only good neighbors are kept in routing tables all routes are established over good quality links. This ensures that both broadcast and unicast messages have equal chances of being received correctly regardless of the used transmission rate. 
Ionut D. Aron et al [6] proposed a localized route repair (LRR) technique, which repairs a route on the fly as soon as it is broken and eliminates the need for network-wide flooding. LRR technique resulted in an enormous reduction in overhead when simulated on an event driven packet level simulator and results in nearly 30 percent overhead savings. This technique improves other network characteristics as well.

Sergio Crisstomo, et. al. proposes an extension to the AODV protocol, denoted Preemptive Local Route Repair (PLRR) [7], that aims to avoid route failures by preemptively local repairing routes when a link break is about to occur. This protocol is to enhance node's information concerning link stability to its neighbours resorting to HELLO messages. These messages are appended with a mobility extension containing the node's position, motion vector and an associated timestamp. This mobility information will be used lo predict the instant $a$ link between two neighbours will break. This proposal does not take into account the sender and destination location information, as other location aided routing protocols. This proposal aims at improving the AODV Quality of Service capabilities by minimizing route failure.

Michael Pan, Sheng-Yan Chuang and Sheng-De Wang proposed an efficient approach to repair error links quickly. As an enhancement to AODV, the proposed approach leads to two routing protocols, called AODV-LRQ and AODV-LRT, which are aimed to efficiently repair the link errors. To evaluate the effects of the route repair, they define a factor, called bonus gain, as the ratio between the throughput increment to the routing overhead increment. Proposed methods can get high bonus gain, that is, it can maintain the throughput as well as reduce the routing overheads.

Ammar Zahary et al, proposed a method [9] called ORMAD (On-demand Route maintenance in Multipath AODV) tries to optimize routing overhead of both Route Discovery Process (RDP) and Route Maintenance Process (RMP) of multipath extensions to AODV. When detecting a link failure in the primary route, ORMAD invokes a local repair procedure between the upstream and the downstream nodes of the broken link. ORMAD applies RMP only to the efficient routes, which leads to enhance route efficiency and consequently minimizes routing overhead. Author shows that the performance is affected by varying the RREP waiting times of both phases RDP and RMP in different scenarios. ORMAD enhances routing packets overhead and average end-to-end delay compared to other protocols, especially in high mobility scenarios.

Nadilma Cintra Valença Nunes Pereira have done, A Comparative Analysis of AODV Route Recovery Mechanisms in Wireless Ad Hoc Networks [10]. This work investigates the behavior of the Ad Hoc On-Demand Distance Vector (AODV) routing protocol in situations of link failures due to the mobility of nodes and consequent route recovery in ad hoc networks. Here, the AODV performance is compared when the failure repair happens locally, at the node before the link break. It was observed that for source repair the packet delivery fraction has better performance, while overhead and delay were better for local repair, in the sparse network and low traffic scenarios studied..

Saaidal R. Azzuhri, et. al, studied that One of the key challenges for routing protocols in wireless multi-hop networks is to deal with link failures, and to repair the routes in these situations. In AODV protocol, routes can either be repaired by re-establishing a new route from scratch starting from the source node (Source Repair), or they can be locally repaired by the node that detects the link break along the end to-end path (Local Repair) [11]. In some situations Source Repair will lead to better performance, in other situations Local Repair will be the more appropriate choice. In this work, author explore a flexible, parameterized approach in deciding on which of these two route repair strategies to use in the event of a link break. It shows that a flexible, parameterised and adaptive approach to choosing the Local Repair Threshold, can improve the Packet Delivery Ratio by up to $37 \%$, compared to the approach employed by standard AODV.

K.A.Shah,M.R.Gandhi demonstrates overall improvement in performance of AODV by Local Route repair. Implementation of Local Route repair reduces the broadcast in case of link outages as in the case of basic AODV [12]. In this scheme data should be buffered in a queue. The node initiates a RREQ and waits for a RREP. If node does not receive any RREP for the destination, it transmits a RERR message to the source node. The source node on receiving the RERR can reinitiate the route discovery if it still desires the route. On the other hand if it receives a one or more RREP during the waiting period, it first compares the hop count of the new route to the previous hop count of the known route, if new hop count is greater than the previously known route hop count then the node send a RERR message for that destination with $\mathrm{N}$ bit set. A node that receives a RERR message with $\mathrm{N}$ flag set, it must not delete the route to that destination.

Jihong Zhao, Rua Qu, Peng Zhang discussed A New Local Repair Scheme for Mobile Ad Hoc Networks. Scheme based on link breaks was proposed to improve route repair efficiency for realistic ad hoc networks [13]. Its design idea depends on the Change of downstream node status is regarded as the basis of choosing route repair schemes by the repairing node. Causes of link breaks would be analyzed according to the change of node status. Different nodes were selected as the destinations for RREQ. RREQ with multiple destinations would be broadcasted by the repairing node. Causes for link breaks are classified as 3 types, i.e, mobility between nodes, node failure and node poweroff. Under different node failure circumstances with the proposed scheme, on average, route setup time is reduced by $13 \%$ and control overhead ratio is lowered by $18 \%$.

\section{REVIEW OF ACO BASED ALGORITHM}

Recently, some ACO-based routing algorithms [14] have emerged for the employment with circuit-switch and voiceapplication networks, wired networks, and in mobile ad-hoc networks. These different biologically-inspired routing algorithms are designed in different ways to satisfy various objectives. Some of the work done in the field of Mobile Ad-hoc network by the researchers is discussed here. Schoonderwoerd et al.[15] designed ABC (Ant-Based Control) for circuit-switched telephone networks. In this method Routing table is maintained by each node. This ACO were able to optimize performance in the network by balancing the load in the network. The AntNet algorithm introduced by Di Caro and Dorigo [16] in 1998 for adaptive routing in packet switching wired networks. AntNet, exhibits some interesting properties: it works in a fully distributed way, is highly adaptive to network and traffic changes, uses lightweight mobile agents (called ants) for active path sampling, is robust to agent failures, provides multipath 
routing, and automatically takes care of data load spreading. Mobile Ants Based Routing (MABR) [17] by M. Heissenbuttel, T. Braun is introduced as the first routing algorithm for MANET's inspired by social insects . In this position-based routing protocols, each node periodically transmits a short hello message (called beacon) to announce its presence and position to update routing table from the corresponding node in highly dynamic networks. The advantages include the ability to react and deal quickly with local and global changes, not only in the network topology, but as well in the communication bandwidth, in the transmission delay, etc. Ant Colony Based Routing (ARA) [18] by Mesut Gunes ,Udo Sorges, Imed Bouazizi gives route discovery mechanism similar to other algorithms such as AODV and DSR. Routes are maintained primarily by data packets as they flow through the network. In the case of a route failure, an attempt is made to send the packet over an alternate link. Otherwise, it is returned to the previous hop for similar processing. Liu Zhenyu in 2005 developed EARA-CG (Emergent Ad hoc Routing Algorithm ) and EARAQoS(Emergent Ad hoc Routing Algorithm )[19] , In these algorithm, the principle of swarm intelligence is used to evolutionally maintain routing information. The core of this QoS provisioning technique is the service class differentiation based queuing scheme. This algorithm provides Congestion control routing. In Ant-based Distributed Routing Algorithm (ADRA) by X. Zheng, W.Guo, and R. Liu [20] 2004, The ants move across the network between randomly chosen pairs of nodes; as they move they deposit simulated pheromones as a function of their distance from their source node, the quality of the link, the congestion encountered on their journey. The ADRA system is shown to result in fewer call failures than the other methods, while exhibiting many attractive features of distributed control. All-Pair ACO by M. T. Islam 2003 [21] was an on-demand routing algorithm called source update for MANET using the ant colony optimization (ACO) search technique. This parallel algorithm were based on source update scales for varying node and machine sizes. This resulted in a good load balance due to the even distribution of work among the ants. AntHocNet by F. Gianni Di Caro, Frederick Ducatelle and Luca Maria GambardellaIn [22]. this paper ACO routing algorithm for MANET is described. It is a hybrid algorithm, combining both proactive and reactive elements: after a reactive route setup phase, the algorithm probes, maintains and improves paths in a proactive way. ARAMA (Ant routing Algorithm for MANET) by O.Hussein 2003[23]. This algorithm is able to fairly distribute the remaining energy across the network nodes and solve the MANETs routing problem under different dynamic conditions.

\section{PROPOSED PROTOCOL WITH NEW ALGORITHM}

Detail study of AODV protocol and research work in the direction of improvement in qualitative parameter give idea for improved protocol. We proposes a modified Ad-hoc on demand distance vector routing protocol. As shown in the figure route discovering method of this protocol is similar to AODV protocol i.e. route discovery is on demand, Managed by RERR and RERP control packets. For the route maintenance during communication a new algorithm is proposed. This algorithm is based on ant colony metaheuristic(figure 3). Our proposed protocol will have following steps:
- In mobile Ad-hoc network all nodes exchange periodic hello message to have information about its neighbors. Nodes also update its neighbors list periodically.

- If any node (Source node) in the network wise to communicate with other node (Destination node) in the network, it look for required node in node list. If this is not available then source node will generate RREQ. Format for route request will be similar as in original AODV.

- When destination node or any node in the link have path up to destination receive route request, it generate route reply (RREP) towards source node. Route reply packet modifies routing table at each node. In our proposed protocol routing table will have one additional column. This column will have IP address of next to next node in the active link, which is modified by RREP packet.

- When source node receive RREP, route for destination is available. Communication is possible from this node to required destination.

- During the communication liveliness of link is assured by periodic hello message. If link break is detected by missing two consecutive hello messages, proposed new algorithm is activated.

- In the case of link failure due to either node movement or power failure or any other reason our local link repairing algorithm is activated. In this algorithm node detecting the link failure will try to repair broken link locally. This node will broadcast artificial ant, forward ant (F-ANT) for the search of next to next node in the link as this information is already available in the modified routing table. At this instant it is considered that after the current link failure rest of the route is still active.

- The format F_ANT is F-ANT<node address,Sequence number (Sn), Destination address, route records (dynamically increasing list, including the intermediate nodes which F-ANT passed through and the corresponding arriving time),Hop count $(\mathrm{Hc})>$

- With this structure forward ant search for next to next node, which is not very far away. In one or two hope this forward ant will reach the required node, having information about the rest of the path.

- Next to next node will generate backward ant (BANT), as this node is having rest of the required active route and data packets can be transferred to destination via this path. BANT structure is < node address, Destination address(the Source address of corresponding $F$-ANT),dynamical route records (its meaning same as in FANT), Sequence number $(S n)>$. During the return path form next to next node to the node generating F-ANT, B-ANT modify route table of intermediate nodes.

- If within the allotted time node requesting the path will not get B-ANT from next to next node. It is assumed that link break can not be mended. In this situation node detecting link break will generate F-ANT for the destination of the required path with timer. Within the allotted waiting time if B-ANT is received from the destination node. Available path can be used for the transmission.

- If this requesting node does not receive any B-ANT within allotted time, It will generated Route error (RRER) message to source node. 


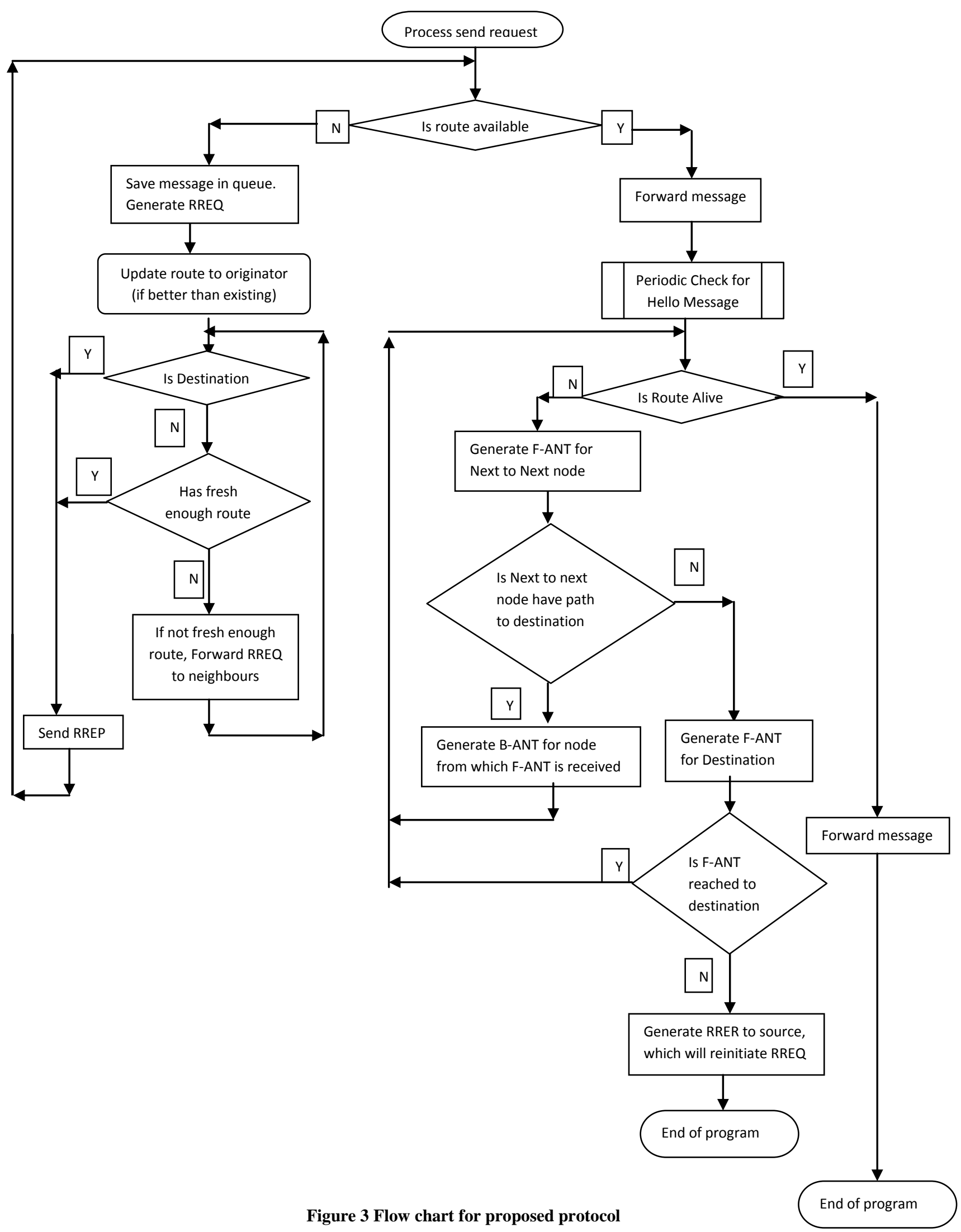




\section{CONCLUSION}

A local repair approach is proposed to enhance the performance of AODV protocol. This algorithm is inspired from the ant colonies meta-heuristic. In case of route failure, forward packets are used to collect information about the network and backward packets are used to update the routing information in the nodes for the local link repairing. With the help of this route repairing mechanism we expect reduction in end to end delay as compared to delay in pure AODV protocol. As in case of route failure alternate route will be available in less time. We also expect increase in throughput as more number of packets can be transmitted successesfully due to availability of alternate route. Overhead in searching re-route will also be reduced, Since route requests are rarer in the case of link breakage.

\section{REFERENCES}

[1] Er J.-N. LIU and Imrich Chlamtac "Mobile ad hoc networking with a view of $4 \mathrm{G}$ wireless: Imperatives and Challenges"

[2] C. S. R. Murthy and B. Manoj "Ad Hoc Wireless Networks: Architectures and Protocols", Prentice Hall PTR, May 2004.

[3] Elizabeth.M.Royer, C-K Toh," A Review of Current Routing Protocols for Ad-hoc Mobile Wireless networks",IEEE personal communication,pp.46-55,April 1999.

[4] C. E. Perkins, E. M. Royer, and S. R. Das, "Ad hoc ondemand distance vector routing," IETF RFC 3561, July 2003.

[5] Srdjan Krco and Marina Dupcinov, "Improved Neighbor Detection Algorithm for AODV Routing Protocol" IEEE Communications letters, Vol. 7, No. 12, December 2003

[6] Ionut D. Aron and Sandeep K.S. Gupta, "Analytical Comparison of local and End-toEnd Error Recovery in Reactive Routing Protocols for Mobile Ad Hoc Networks," Proceedings of the 3rd ACM Int. Workshop on Modeling, Analysis and Simulation of Wireless and Mobile Systems (MSWIM 2000) pp. 69-76, August 2000.

[7] Sergio Crisstomo , Susana Sargento, edro Brandgo', Rui Prior' "Improving AODV with Preemptive Local Route Repair" International Workshop on Wireless Ad-Hoc Networks 2004

[8] XIAO Bai-Long, GUO Wei, LIU Jun, ZHU Si-Lu, “An Improvement for Local Route Repair in Mobile Ad Hoc Networks," 6th International Conference on ITS Telecommunications Proceedings 2006.

[9] Ammar Zahary and Aladdin Ayesh , "On-demand Multiple Route Maintenance in AODV Extensions (ORMAD)" IEEE page 225-230, 2008.

[10] Nadilma Cintra Valença Nunes Pereira and Renato Mariz de Moraes, " A Comparative Analysis of AODV Route Recovery Mechanisms in Wireless Ad Hoc Networks" IEEE transaction 2009
[11] Saaidal R. Azzuhri, Marius Portmann, Wee Lum Tan, "Evaluation of Parameterised Route Repair in AODV" IEEE 2010.

[12] S. H. Shah and K. Nahrsledt.. "Predictive location-based QoS routing in mobile ad hoc networks," in in Pruc. of IEEE Infernarional Conferenceon Comniunicarions (ICC 20021, New York, NZ April - May 2002

[13] Fei Jiang, JianJun Hao, "Simulation of An Improved AODV Algorithm for Ad Hoc Network" voll IEEE conference ICACT 2010 ,

[14] [14] M.Dorigo, LM. Gambardella, "Ant colony system: a cooperative learning approach to the traveling salesman problem", IEEE Transactions on Evolutionary Computation, Vol.1, No.1, pp.53-66,1997.

[15] Ruud Schoonderwoerd, Owen Holland, Janet Bruten, and L.Rothkrantz, "Ant-Based Load Balancing In Telecommunications Networks," Adaptive Behavior, 1996.

[16] G.Di Caro and M.Dorigo, "AntNet: distributed stigmergetic control for communications networks," Journal of Artificial Intelligence Research, vol. 9, pp. 317-365, 1998.

[17] M.Heissenbuttle and T.Braun, "Ants-Based Routing in Large Scale Mobile Ad-Hoc Networks," Kommunikation in Verteilten Systemen (KiVS), 2003.

[18] Mesut Gunes,Udo Sorges, Imed Bouazizi, "ARA-The AntColony Based Routing Algorithm for MANETs" International workshop on Ad HocNetworking (WAHN 2002) couver, British Columbia, Canada, August1821,2002

[19] Zhenyu Liu; Marta Z. Kwaitkowska; Costas Constantinou "A Biologically Inspired QoS Routing Algorithm for Mobile Ad Hoc Networks", Proceedings of the 19thInternational Conference on Advanced Information. Networking and Applications(AINA'05), 2005. pp. 426 431

[20] X. Zheng, W. Guo, and R. Liu. An ant-based distributed routing algorithm for ad-hoc networks. In Proceedings of the International Conference on Communications, Circuits

and Systems (ICCCAS), pages 412-417, Washington,DC, 2004. IEEE.

[21] M. T. Islam, P. Thulasiraman, and R. K. Thulasiram, "A parallel ant colony optimization algorithm for all-pair routing in MANETs," in Proceedings of the International Symposium on Parallel and Distributed Processing (IPDPS 2003), Nice, France, April 2003.

[22] Gianni Di Caro and Gianni Di Caro and Frederick Ducatelle and Frederick Ducatelle and Luca Maria Gambardella and Luca Maria Gambardella, "AntHocNet: An Adaptive Nature-inspired Algorithm for Routing in Mobile Ad hoc Networks", European Transactions on

Telecommunications, vol. 16, pp. 443-455, 2005.

[23] O. Hussein, T. Saadawi, "Ant routing algorithm for mobile ad-hoc networks (ARAMA)", performance computing, and communications IEEE International conference, pp. 281290, 2003. 